\title{
Developing New Measurement Capabilities with Nanochannel Liquid Phase TEM
}

Murat Nulati Yesibolati ${ }^{1}$, Hongyu Sun ${ }^{1}$, Silvia Canepa ${ }^{1}$, Simone Lagana ${ }^{1,2,3}$, Takeshi Kasama ${ }^{2}$, Shawn Kathmann ${ }^{4}$, Damien Alloyeau ${ }^{5}$, Kristian Mølhave ${ }^{1}$

1. Dept. of Micro- and Nanotechnology, Technical University of Denmark (DTU), Lyngby, Denmark.

2. Center for Electron Nanoscopy, Technical University of Denmark (DTU), Lyngby, Denmark.

3. Dept. of Chemistry, Technical University of Denmark (DTU), Lyngby, Denmark.

4. Physical Sciences Division, Pacific Northwest National Laboratory, WA, USA

5. Laboratoire Matériaux et Phénomènes Quantiques, Université Paris Diderot, France

By creating new designs of liquid phase transmission electron microscopy (TEM) systems we develop new capabilities for measurements of physical and chemical processes. A microfabricated chip with suspended nanochannels $[1,2]$ provide high resolution imaging possibilities, direct TEM imaging of flow in nanochannels, and also makes electron holographic TEM possible [3].

Imaging a nanochannel by TEM holography makes it possible to perform quantitative measurements of the electron phase shift through the liquid medium and immersed samples, Fig 1. We have measured the mean inner potential of water and immersed nanoparticles. Preliminary analysis indicate the mean inner potential of liquid water is $4.3 \pm 0.6 \mathrm{~V}$, somewhat above the only other experimental value based on vitrified ice of $3.5 \pm 1.3 \mathrm{~V}[4]$.

When bubbles expand in the channel system, free nanoparticles are moving with the liquid flow, Fig 2. Mapping the particle velocity profile gives a position dependence of flow velocity matching that of parabolic Poiseuille Flow in the observed nanochannel geometry. As the nanochannel system makes it possible to follow the detailed dynamics of nanoparticle motion and agglomeration in a confined system, the commercially available systems with clamped membrane chips also allow detailed studies of diffusion processes. Numerous papers have reported highly limited diffusion in liquid phase TEM of particles in close contact with the membrane, and we have made a detailed study of the Brownian motion of the sub-population of fast and freely moving particles, Fig 3. Their diffusion is Brownian with Gaussian step length distribution, and the diffusion coefficient can be matched with the Faxén correction to diffusion near surfaces, but is also influenced by the STEM beam current.

The nanochannel system provides an interesting confined geometry for new types of experiments studying nanoscale fluidics and interactions, where also the potential mapping capabilities of holographic TEM is possible [5].

References:

[1] S Laganá et al, Microelectronic Engineering 176 (2017), p. 71-74,

[2] E. Jensen, A. Burrows, and K. Mølhave, Microsc. Microanal., 20(02) (2014), p. 445.

[3] M. N. Yesibolati, et al, Microsc. Microanal., 22(S5) (2016), pp. 86.

[4] A Harsher and H Lichte, Electron Microscopy 1 (1998), p. 553.

[5] The authors acknowledge funding DTU and from the Danish Research Council for Technology and Production Case No. 12-126194. S.M.K. was supported by the U.S. Department of Energy, Office of Science, Office of Basic Energy Sciences, Division of Chemical Sciences, Geosciences and Biosciences. Pacific Northwest National Laboratory (PNNL) is a multiprogram national laboratory operated for DOE by Battelle. 

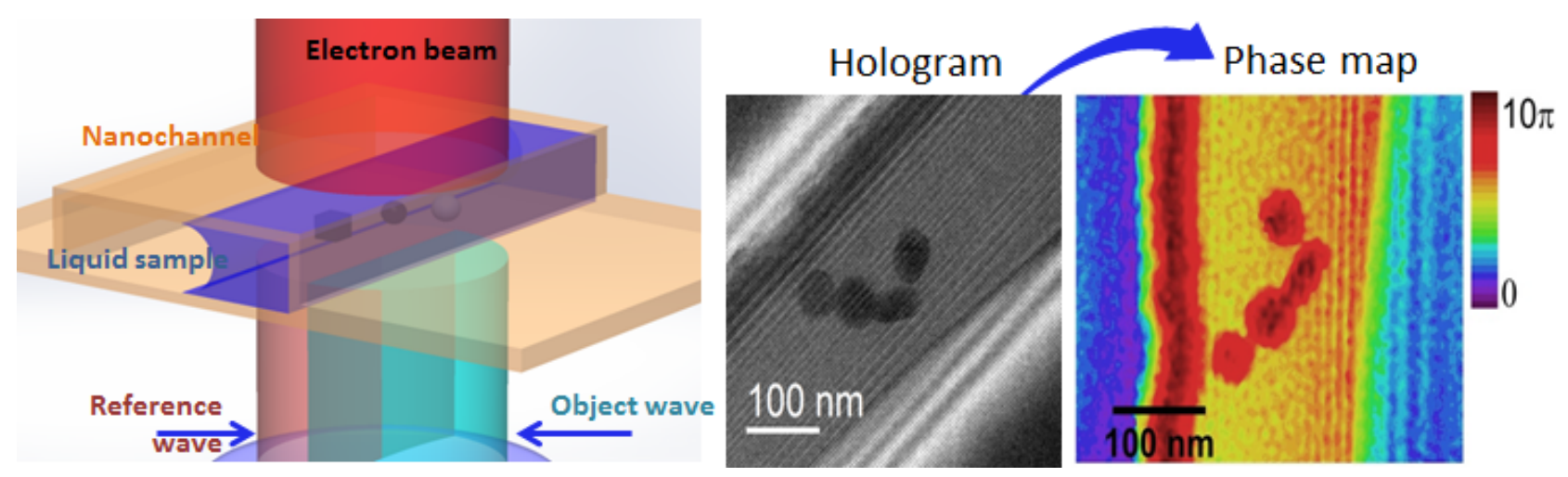

Figure 1. Electron holographic imaging of liquid samples in a suspended nanochannel gives a holographic image that can provide a phase map of the liquid sample.
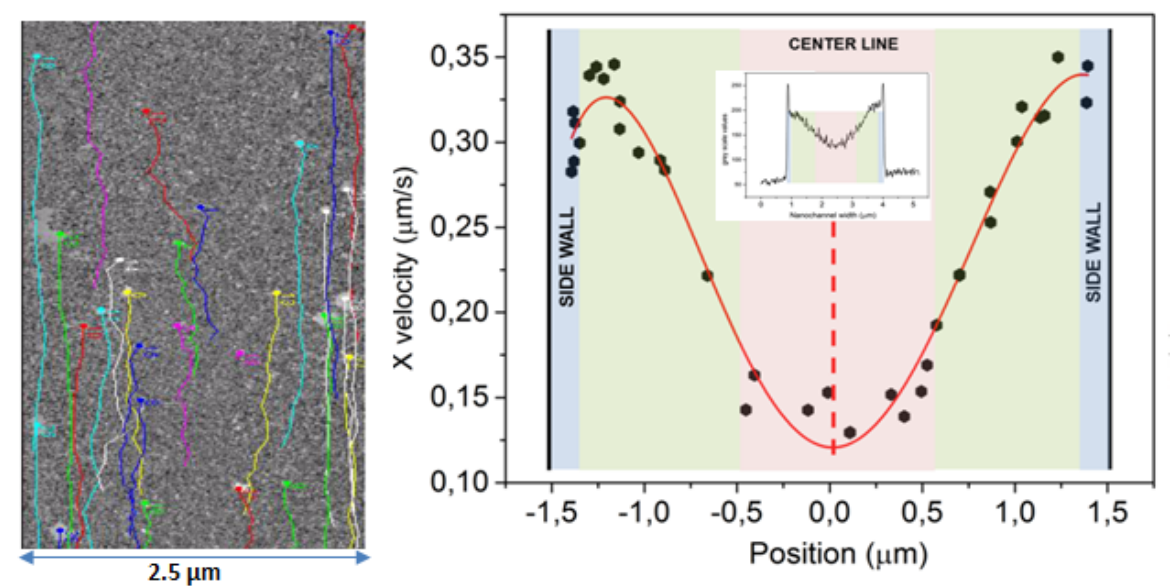

Figure 2. Flow in nanochannel observed by STEM, and tracked by subtracting the first image to remove background. The channels have an inwards deflection and hence a thinner liquid layer at the center of the channel as indicated by the insert showing a STEM signal profile across the channel. The measured flow velocities match a parabolic flow profile with lower flow velocities at the center and also lower near the channel walls.
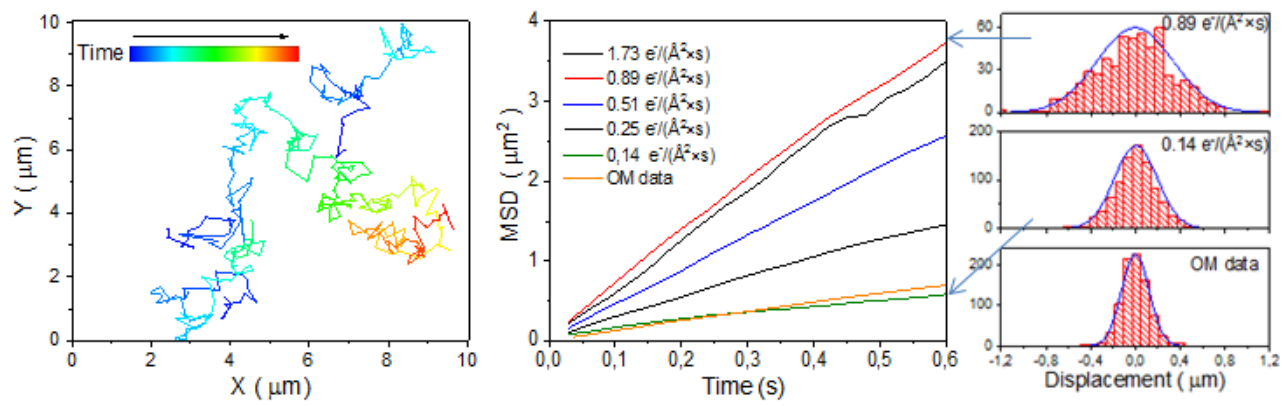

Figure 3. Brownian motion of a freely moving gold nanoparticle in a liquid cell. Mean square displacement and step length histogram indicate Brownian motion with a diffusion coefficient depending on the beam current density. 